\title{
Morphology and the morphometric measurements of hypertensive and normotensive placenta.
}

\author{
Akshara VR ${ }^{*}$, Ramakrishnan PK ${ }^{1}$, Chitra $S^{2}$, Kumar Sai Sailesh ${ }^{3}$, Senthil Kumar $S^{4}$, Seema Valsalan \\ $\mathbf{E}^{1}$ \\ ${ }^{1}$ Department of Anatomy, PK Das Institute of Medical Sciences, Ottapalam, Kerala, India \\ ${ }^{2}$ Department of pathology, Saveetha Medical College and Hospital, Saveetha Institute of Medical and Technical \\ Sciences, Chennai, Tamil Nadu, India \\ ${ }^{3}$ Department of Physiology, Vishnu Dental College, Vishupur, Bhimavaram, West Godavari District, Andhra Pradesh, \\ India \\ ${ }^{4}$ Department of Anatomy, SRMC, Porur, Chennai, Tamil Nadu, India
}

\begin{abstract}
The placenta is a feto-maternal organ which has a fetal part develops from the chorionic sac which is lined by amnion and completely covered by chorion with an attachment of the umbilical cord and maternal part from the endometrium. The current study was aimed to analyse the morphology and the morphometric measurements of hypertensive and normotensive placenta. Placentas of $\mathbf{4 0}$ hypertensive and 46 healthy women who attended antenatal clinic of the hospital regularly and delivered their babies in the same hospital were collected. Weight, diameter, thickness, surface area, volume and circumference, shape, umbilical cord insertion and membrane attachment of the placenta was assessed using standard methods mentioned in the literature. There is no significant differences present among the morphometric parameters of placenta. The study recommends further detailed and multi centered studies in this area to understand the correlation to plan better management programs for the benefit of pregnant women. We recommend further detailed studies in this area for better understanding of the condition and to plan adequate management methods for the benefit of women in general.
\end{abstract}

Keywords: Placenta, Morphometric measurements, Hypertension.

Accepted on October 9, 2018

\section{Introduction}

The placenta is a feto-maternal organ which has a fetal part develops from the chorionic sac which is lined by amnion and completely covered by chorion with an attachment of the umbilical cord and maternal part from the endometrium. The transfer of nutrients and the gaseous exchange between the mother and the fetus takes place in the placenta. It is one of the fascinating organ having wide range functions including protection, nutrition, respiration, excretion and hormone production [1]. Human placenta is defined as haemochorial and deciduate. It is a flattened discoid mass with an approximately oval or circular in outline with an average volume of $500 \mathrm{ml}$ (200-950 ml), average weight about $500 \mathrm{~g}(200-800 \mathrm{~g})$, average diameter $185 \mathrm{~mm}(150-200 \mathrm{~mm})$, average thickness 23 $\mathrm{mm}(10-40 \mathrm{~mm})$ and an average surface area about $(30000$ $\mathrm{mm}^{2}$ ) and thickest at its center [2]. Intrauterine existence of the fetus depends on the proper development of placenta. Improper development of this structure will have an impact on the fetus and also results in complications like maternal hypertension, gestational diabetes, IUGR etc. Sometimes idiopathic intrauterine growth restricted babies can be the result of pregnancy but there will be no specific complications in that case placental examination will provide an insight into that specific condition. Hypertension is one of the common complications met with in pregnancy and diagnosed if maternal blood pressure is more than $140 / 90 \mathrm{~mm} \mathrm{Hg}$. Hypertension in pregnancy may be chronic hypertension (onset before pregnancy) or may be induced due to pregnancy like, gestational hypertension, preeclampsia and eclampsia. In hypertension, there will be decrease in the blood supply due to vasoconstriction of the maternal blood vessels [3]. These complications of hypertension alter the architecture of placenta. Careful examination will provide an accurate account of in utero events of fetus. Prenatal experiences of mother and baby can be diagnosed by the examination of placenta. Placental barrier is protective for the baby against infections and harmful substances Growth of fetus depend upon the morphology, metabolic, immunological, endocrine functions and blood flow of placenta. Many of the complications will be 
reflected in the gross architecture of the placenta [4]. That one minute examination is so important. This will protect obstetricians, pediatricians and surgeons from the medico legal issues if there is any poor maternal or fetal outcome and also help them in the management of the successive pregnancies and the infants. The current study was aimed to analyse the morphology and the morphometric measurements of hypertensive and normotensive placenta [5-14].

\section{Materials and Methods}

\section{Study design: case control study}

Study setting: The study was conducted at Department of Anatomy and Department of Obstetrics and Gynaecology of P. K. Das Institute of Medical Sciences, Ottapalam, Palakkad, Kerala, India.

Study participants: Placentas of 40 hypertensive and 46 healthy women who attended antenatal clinic of the hospital regularly and delivered their babies in the same hospital were collected. Written Informed consent was obtained from all the participants after explaining the study in detail and ensuring the confidentiality. Complete medical, social and obstetric history was recorded to identify the confounders. The mothers were examined clinically along with recording of relevant investigation reports. All control subjects had no history of raised blood pressure at any stage during their life and had no evident hypertension and proteinuria during pregnancy. The following criteria were used to recruit the cases.

Inclusion criteria: Hypertensive women who: Had hypertension in pregnancy after the $20 \mathrm{w}$ of gestation, Had consistently recorded SBP and DBP of 140 and $90 \mathrm{~mm} \mathrm{Hg}$ respectively with or without edema and/or proteinuria were included.

Exclusion criteria: Women having any other serious diseases like endocrine disorders, cardio vascular diseases, respiratory diseases, renal diseases. Diabetes mellitus, anemia were excluded from the study.

Placental parameter: Weight, diameter, thickness, surface area, volume and circumference, shape, umbilical cord insertion and membrane attachment of the placenta was assessed using standard methods mentioned in the literature. The placenta with cords and membranes were collected and observed immediately after the delivery. Any abnormality of the umbilical cord and membrane was recorded. In all these cases, the amnion and chorion were trimmed off from the placenta. The umbilical cord was cut at a distance of $10 \mathrm{~cm}$ from the site of insertion. Placentae were washed in slow running tap water dried with the help of blotting paper. The placentae were weighed with a standard weighing machine. The shape of the placenta and presence of accessory lobe were recorded after proper inspection. Each placenta was categorized as oval, circular, triangular or irregular in shape. Diameter was measured by placing the placenta in a flat tray; the first maximum diameter was measured by a metallic scale graduated in centimeters $(\mathrm{cm})$. Then second maximum diameter was taken at right angles to the first one. The mean of two measurements was considered as the diameter of the placenta expressed in centimeters. The thickness of the placenta was measured by inserting a calibrated Knitting needle at the center of placenta and measured in centimeter each placenta was placed on fetal surface. The maternal surface area of the placenta was calculated using the following formula: Surface area $=\pi \mathrm{d} 1 \times \mathrm{d} 2 / 4$, (where $\mathrm{dl}$ is the largest diameter and ds are the smallest diameter. Circumference is calculated by formula $\pi \times \mathrm{d}$. Volume is calculated by formula $\pi r^{2} h$.

Ethical consideration: The study protocol was approved by institutional human ethical committee of P.K. Das Institute of Medical Sciences, Kerala, India. (CRF/CRL/P11-1/Ph.D).

Data analysis: Data was analysed using SPSS 20.0. Statistical test used are unpaired t test. Data was presented as mean and standard error. $\mathrm{P}$ value less than 0.05 was considered as significant.

\section{Results}

Figure 1 presents the demographic data of the participants. Age and height are not significantly different between normotensive and hypertensive patients. Significantly higher weight $(\mathrm{P}<0.01)$ and $\mathrm{BMI}(\mathrm{P}<0.05)$ was observed in hypertensive women when compared to normotensive. Figure 2 represents weight, thickness, diameter and surface area of the placenta. These parameters are not significantly different between normotensive and hypertensive placenta Figure 3 represents circumference, volume, FP ratio and placental coefficient which are not significantly different between normal and hypertensive group.
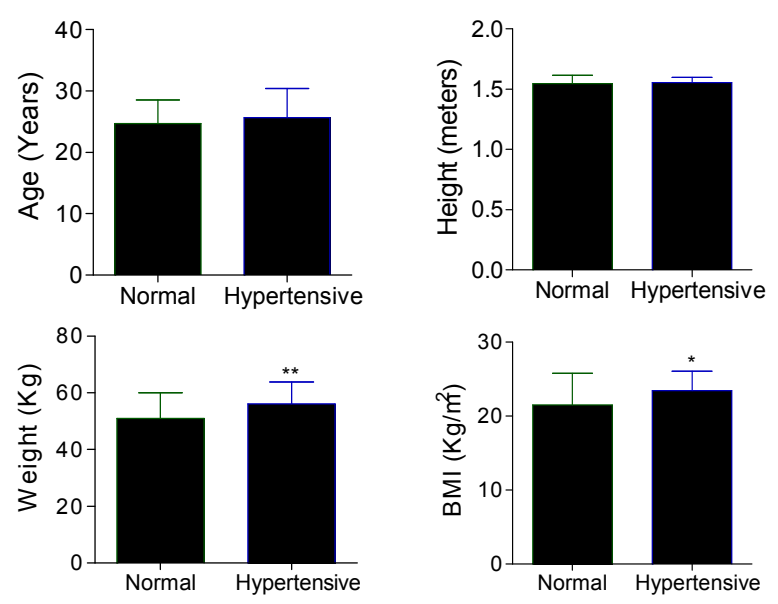

Figure 1. Demographic data of the participants (Data was presented as mean $\pm S E)\left({ }^{*} P<0.05\right.$ was significant, ${ }^{* *} P<0.01$ was significant $)$.

Table 1 shows the frequency distribution of morphology of placenta such as shape, cord insertion and membrane attachment. Regarding the shape in normal placentas $60.8 \%$ are round, 34.7\%oval and 4.34\% are irregular. Among hypertensive placenta $52 \%$ are round, $30 \%$ are oval, $12.5 \%$ irregular, $5 \%$ triangular in shape. Central insertion of cord was 
shown by $47.82 \%$ in normal and $37.5 \%$ in hypertensive placentas, eccentric insertion among normal is $39.13 \%$ and 55 $\%$ in hypertensive group, marginal insertion in $4.34 \%$ in normal and 5\% in hypertensive group, velamentous insertion shown by $4.34 \%$ in normal group and no cases in hypertensive group, furcated insertion shown by $4.34 \%$ in normal group and $5 \%$ in hypertensive group. Regarding the membrane attachment $84.78 \%$ showed normal attachment, $13.4 \%$ circummarginate and $2.17 \%$ showed circumvallate attachment.IN hypertensive group $65 \%$ having normal membrane attachment, $17.5 \%$ showed circummarginate and $17.5 \%$ showed circum vallate attachment of membrane.
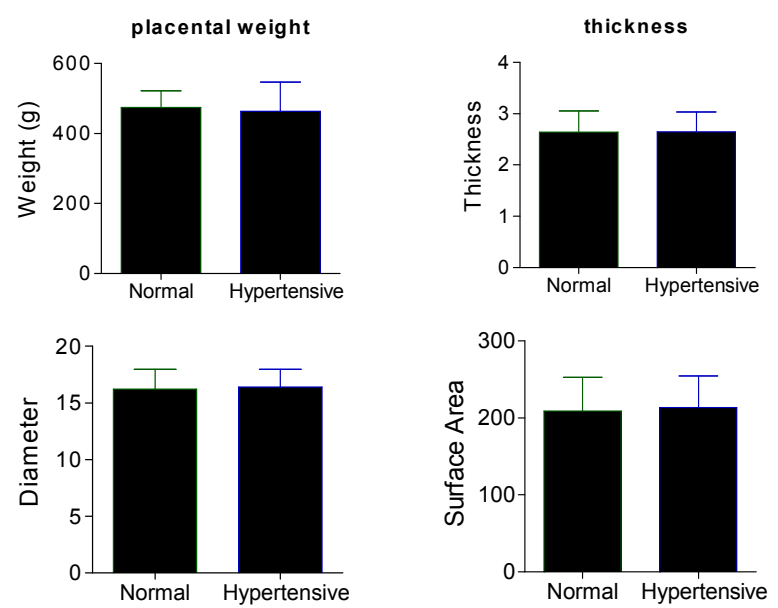

Figure 2. Placental weight, thickness, diameter and surface area of normotensive and hypertensive participants. (Data was presented as mean $\pm S E)\left({ }^{*} P<0.05\right.$ was significant, ${ }^{* *} P<0.01$ was significant $)$.

Table 1. Placental morphology of normotensive and hypertensive participants. (Data was presented as frequency and percentage).

\begin{tabular}{llll}
\hline Parameter & \multicolumn{1}{l}{ Type } & Normotensive & Hypertensive \\
\hline Shape of placenta & Round & $28(60.8 \%)$ & $21(52 \%)$ \\
\cline { 2 - 4 } & Oval & $16(34.7 \%)$ & $12(30 \%)$ \\
\cline { 2 - 4 } & Triangle & $0(0 \%)$ & $2(5 \%)$ \\
\cline { 2 - 4 } & Irregular & $2(4.34 \%)$ & $5(12.5 \%)$ \\
\hline Cord insertion & Marginal & $2(4.34 \%)$ & $2(5 \%)$ \\
\cline { 2 - 4 } & Central & $22(47.82 \%)$ & $15(37.5 \%)$ \\
\cline { 2 - 4 } & Eccentric & $18(39.13 \%)$ & $22(55)$ \\
\cline { 2 - 4 } & Velementous & $2(4.34 \%)$ & $0(0 \%)$ \\
\cline { 2 - 4 } & Furcate & $2(4.34 \%)$ & $2(5 \%)$ \\
\hline Membrane & Normal & $39(84.78 \%)$ & $26(65 \%)$ \\
\cline { 2 - 4 } & Circum marginate & $6(13.04 \%)$ & $7(17.5 \%)$ \\
\cline { 2 - 4 } & Circumvallate & $1(2.17 \%)$ & $7(17.5 \%)$ \\
\hline
\end{tabular}

\section{Discussion}

Placenta is a specialized structure as part of it is made from mother and other part from the fetus. The placenta is an important structure which connects the fetus with the wall of the uterus. This helps for transportation of the nutrients and other substances. As the morphology of placenta was complex, it was suggested to do quantitative analysis of placenta than qualitative [15-17]. The current study was undertaken to analyse the morphology and the morphometric measurements of hypertensive and normotensive placenta. There was no difference in age and height parameters but the weight of hypertensive was found to be higher than normotensive. Morphological features are not significantly different between the normotensive and hypertensive placenta. The circumference, volume, FP ratio and placental coefficient are not significantly different between normal and hypertensive group. The placental examination provides better understanding of the prenatal health of both the child and mother [7]. The complications of the pregnancy like hypertension, diabetes are well represented in the placenta [8]. Earlier studies reported that there is significant decrease in the placental surface area in hypertension [8,9]. This may leads to decrease in the blood and oxygen supply to fetus and leads to death of the fetus. It was reported that the morphometric parameters like weight, surface area, volume etc. were significantly decreased in the hypertensive [10-14].
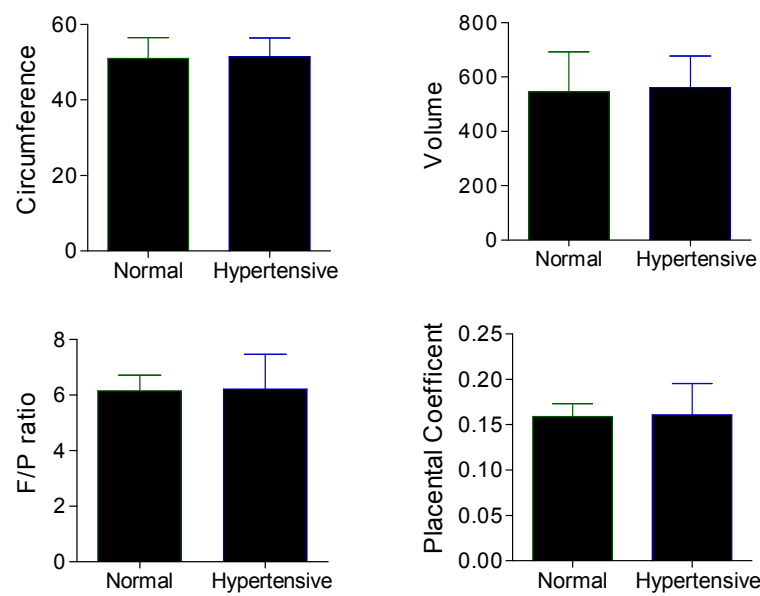

Figure 3. Placental circumference, volume, placental coefficient and FP (fetal and placental weight) ratio. (Data was presented as mean \pm SE) $\left({ }^{*} P<0.05\right.$ was significant, ${ }^{* *} P<0.01$ was significant $)$.

Earlier studies reported that majority of the placenta was oval shape in both normotensive and hypertensive [18]. In another study from Pakistan, it was observed that the shape was both round and oval [19]. In the current study majority of placenta is round shape in both the groups. Earlier studies reported that marginal insertion was present in majority of placentae of hypertensive [20]. In the current study, the eccentric insertion was observed in majority of hypertensive placenta and central insertion in majority of normotensive. The membrane of placenta was normal in the present study in both the groups.

\section{Conclusion}

There is no significant differences present among the morphometric parameters of placenta. The study recommends 
further detailed and multi centered studies in this area to understand the correlation to plan better management programs for the benefit of pregnant women. We recommend further detailed studies in this area for better understanding of the condition and to plan adequate management methods for the benefit of women in general.

\section{Limitations}

The study was conducted at one center only. So the results cannot be generalized.

\section{References}

1. Lacroix M, Kina E, Hivert MF. Maternal/fetal determinants of insulin resistance in women during pregnancy and in offspring over life. Curr Diab Rep 2013; 13: 238-244.

2. McMaster MT, Zhou Y, Fisher SJ. Abnormal placentation and the syndrome of preeclampsia. Semin Nephrol 2004; 24: $540-547$.

3. Fisher SJ. The placental problem: linking abnormal cytotrophoblast differentiation to the maternal symptoms of preeclampsia. Reprod Biol Endocrinol 2004; 2: 53.

4. Imogen P, Anna S, Ainslie G, Sian B, Nicola B, Gauri B, Colin PS, Rebecca LJ, Paul B, Alexander EPH. Quantitative assessment of placental morphology may identify specific causes of stillbirth. BMC Clin Pathol 2016; 16: 1 .

5. [No authors listed]. ACOG Practice Bulletin No. 102: management of stillbirth. Obstet Gynecol 2009; 113: 748-761.

6. Royal College of Obstetricians and Gynaecologists. Green-Top Guideline 55-Late Intrauterine Fetal Death and Stillbirth. London: Royal College of Obstetricians and Gynaecologists 2010.

7. Bewley S, Cooper D, Campbell S. Doppler investigation of uteroplacental blood flow resistance in the second trimester: a screening study of or pre-eclampsia and intrauterine growth retardation. Br J Obstet Gynaecol 1991; 98: 871-879.

8. Browne JC, Veall N. The maternal placental blood flow in normotensive and hypertensive women. J Obstet Gynaecol Br Emp 1953; 60: 141-147.

9. Rath G, Garg K. Vascular pattern of human placenta in complicated pregnancy, a corrosive cast study. Ann Nat Acad Med Sc (Ind)1994; 30: 17-22.
10. Stock MK, Anderson DF, Phernetton TM. Vascular response of the fetal placenta to local occlusion of the maternal placental vasculature. J Dev Physiol 1980; 2: 339-346.

11. Udainia A, Bhagwat SS, Mehta CD. Relation between placental surface area, infarction and foetal distress in pregnancy induced hypertension with its clinicalrelevance. J Anat Soc Ind 2004; 53: 27-30.

12. Naeye RL, Friedman EA. Causes of perinatal death associated with gestational hypertension and proteinuria. Am J Obstet Gynecol 1979; 133: 8-10.

13. Fox H. The placenta in intra uterine growth retardation. Early foetal growth and development. RCOG Press London 1994; 223-235.

14. Nag U, Chakravarthy VK, Rao DR. Morphological changes in placenta of hypertensive pregnant women. IJRRMS 2013; 3: 1-4.

15. Teasdale F. Gestational changes in the functional structure of the human placenta in relation to fetal growth: a morphometric study. Am J Obstet Gynecol 1980; 137: 560-563.

16. Benirschke K, Burton GJ, Baergen RN. Pathology of human placenta (6th Edn.). New York Springer 2012.

17. Fox H, Neil J. Pathology of the edition. Philadelphia: Elsevier Saunders 2007.

18. Gyan CA, Pankaj S, Jai PP, Anjali J. Morphological study of placenta in normal and hypertensive pregnancies. Int Arc Integr Med 2015; 5: 121-128.

19. Ashfaq M, Janjua MZ, Channa MA. Effect of gestational diabetes and maternal hypertension on gross morphology of placenta. J Ayub Med Coll Abbottabad 2005; 17: 44-47.

20. Rath G, Garg K, Sood M. Insertion of umbilical cord on the placenta in hypertensive mother. Anat Soc India 2000; 49: 149-152.

\section{*Correspondence to}

Akshara VR

Department of Anatomy

PK Das Institute of Medical Sciences

Kerala

India 